\title{
Syncope in patients with drug-resistant epilepsy without apparent cardiovascular disease
}

Síncope em pacientes com epilepsia fármaco resistente sem doença cardiovascular aparente

Soniza Vieira Alves-Leon 1,2, Moisés Pereira Pinto', Maria Emilia Cosenza Andraus ${ }^{3}$, Valéria Coelho Santa Rita Pereira', Isabella D'Andrea Meira', Raquel de Carvalho Oliveira', Shaylla Villas Boas ${ }^{1}$, Cláudia Cecilia da Silva Rêgo ${ }^{1}$, Jorge Paes Barreto Marcondes de Souza ${ }^{1,4}$, Roberto Coury Pedrosa ${ }^{5}$

\begin{abstract}
Epilepsy and syncope are clinical conditions with high prevalence rates in the general population, and the differential diagnosis between them is difficult. Objective: To assess the frequency of syncope in patients diagnosed with drug-resistant epilepsy (DRE) without apparent heart disease, to investigate the relationship between clinical and electroencephalographic (EEG) changes, and to verify the role of the inclination test (IT). Method: An open, prospective study from 2004 to 2006, including 35 consecutive patients from the Epilepsy Program of Hospital Universitário Clementino Fraga Filho who were diagnosed with DRE without apparent heart disease. Results: The frequency of syncope was $25.7 \%(n=9)$, with a significant prevalence in women. Vasovagal syncope (VVS) was the most frequent diagnosis. Conclusion: We found a significant association between syncope and the presence of autonomic symptoms ( $p=0.005)$. The IT plays an important role in the differential diagnosis of patients with DRE presenting with autonomic symptoms, regardless of EEG results and brain magnetic resonance imaging (MRI) abnormalities.
\end{abstract}

Keywords: drug-resistant epilepsy, epilepsy, inclination test, syncope, pseudoseizures.

\section{RESUMO}

Epilepsia e síncope são condições clínicas com alta prevalência na população geral e, às vezes, o diagnóstico diferencial entre elas é difícil. Objetivo: Investigar a frequência de síncope em pacientes diagnosticados com epilepsia fármaco resistente (EFR), sem doença cardíaca aparente; investigar a relação entre alterações clínicas e eletrencefalográficas; verificar o papel do teste de inclinação (TI). Método: Estudo aberto prospectivo, realizado de 2004 a 2006, incluindo 35 pacientes consecutivos do Programa de Epilepsias do Hospital Universitário Clementino Fraga Filho, diagnosticados com EFR, sem doença cardíaca aparente. Resultados: A frequência de síncope foi de 25,7\% ( $n=9$ ), com prevalência significativa em mulheres. Síncope vasovagal (SVV) foi o diagnóstico mais frequente. Conclusão: Encontramos uma significativa associação entre síncope e a presença de sintomas autonômicos ( $p=0,005)$. O TI tem importante papel no diagnóstico diferencial de pacientes com diagnóstico de EFR que apresentam sintomas autonômicos, a despeito de alterações eletrencefalográficas e de ressonância magnética do crânio.

Palavras-chave: epilepsia fármaco-resistente, epilepsia, teste de inclinação, pseudocrises.

It is estimated that $0.5-1.5 \%$ of the general population has epilepsy, and approximately $5 \%$ of people experience one or more seizures during their lifetime ${ }^{1}$. Epilepsy is one of the most prevalent neurological conditions worldwide, mainly in developing countries ${ }^{1,2}$. Proper treatment depends on the correct diagnosis. Drug-resistant epilepsy (DRE) affects an estimated $30 \%$ of patients with epilepsy ${ }^{3}$ and approximately 500,000 people in $\mathrm{Brazil}^{4}$. DRE is considered when the patient

1Programa de Epilepsia, Departamento de Neurologia, Hospital Universitário Clementino Fraga Filho, Universidade Federal do Rio de Janeiro, Rio de Janeiro RJ, Brazil;

${ }^{2}$ Professor Associado de Neurologia, Programa de Pós-Graduação, Universidade Federal do Estado do Rio de Janeiro, Rio de Janeiro RJ, Brazil;

${ }^{3}$ Serviço de Neurologia Prof. Sérgio Novis, Santa Casa da Misericórdia do Rio de Janeiro, Rio de Janeiro RJ, Brazil;

${ }^{4}$ Departamento de Neurocirurgia, Hospital Universitário Clementino Fraga Filho, Professor de Neurocirurgia, Universidade Federal do Rio de Janeiro, Rio de Janeiro RJ, Brazil;

${ }^{5}$ Departamento de Cardiologia, Hospital Universitário Clementino Fraga Filho, Universidade Federal do Rio de Janeiro, Rio de Janeiro RJ, Brazil.

Correspondence: Soniza Vieira Alves-Leon; Programa de Epilepsias / Hospital Universitário Clementino Fraga Filho, UFRJ; Unidade de Vídeo-EEG Enfermarias 10C2 e 10C4; Rua Prof. Rodolpho Paulo Rocco 255 / Cidade Universitária; 21941-617 Rio de Janeiro RJ - Brasil; E-mail: sonizavleon@globo.com

Support: Fundação de Amparo à Pesquisa do Estado do Rio de Janeiro (FAPERJ), Conselho Nacional de Desenvolvimento Científico e Tecnológico (CNPq).

Conflict of interest: There is no conflict of interest to declare.

Received 25 February 2013; Received in final form 09 July 2013; Accepted 16 July 2013. 
has recurrent seizures, despite the administration of two antiepileptic drugs (AEDs) as monotherapy or in combination at the maximum permitted levels, with proven treatment adherence ${ }^{5}$. DRE is a serious and limiting condition that dramatically affects the independent living of the patients ${ }^{3}$.

Syncope is one of the paroxysmal clinical events considered in the differential diagnosis of epilepsy, especially DRE. It is defined as episodes of transient loss of consciousness, with relatively sudden onset and loss of postural tone, followed by spontaneous and complete recovery, without persistent neurological signs, that does not require chemical or electrical cardioversion ${ }^{6,7}$. It is estimated that $20-30 \%$ of patients with epilepsy have other causes for loss or disturbance of consciousness, even those monitored in centers for epilepsy treatment ${ }^{8}$. These non-epileptic events may occur in up to $45 \%$ of patients treated in epilepsy program centers ${ }^{5,9}$.

The purpose of this study was to investigate the frequency of syncope and its associated variables among patients diagnosed with DRE without apparent heart disease, to investigate the relationship between clinical and electroencephalographic changes, and to verify the role of the inclination test (IT) in the investigation of patients diagnosed with DRE who present with autonomic dysfunction.

\section{METHOD}

We included consecutive patients over 16 years old (both male and female) from the Epilepsy Program of Hospital Universitário Clementino Fraga Filho/Universidade Federal do Rio de Janeiro (HUCFF/UFRJ) who met criteria for the diagnosis of DRE independent of the association with clinical manifestations suggestive of autonomic dysfunction. Ictal phenomenology could include primary generalized seizures (PGS) or focal onset with secondary generalized seizures (SGS) according to the Engel classification ${ }^{10}$. The selected patients had normal cardiac evaluations, which included anamnesis, physical examination, electrocardiogram (EKG), echocardiogram (ECHO), 24-hour Holter monitoring, and ambulatory blood pressure monitoring (ABPM).

We excluded patients with focal onset seizures who had no secondary generalization, even with DRE; patients with heart disease symptoms; patients with mental retardation or physical problems that prevented them from standing during the IT; patients with irregular heart rate; those for whom carotid sinus massage was associated with some risk (including patients with carotid bruit); and those who did not consent to participate. There were 412 enrolled patients in the HUCFF/UFRJEpilepsy Program, and 296 (71.8\%) of them met the diagnostic criteria for DRE. After application of the inclusion and exclusion criteria, we selected 39 consecutive patients with DRE presenting with PGS or SGS and an absence of clinical symptoms of cardiac disease. Of these 39 patients,
4 were excluded owing to suspected heart disease after review, and a total of 35 patients were included in the study.

All included patients signed an informed consent form and were enrolled consecutively from July 2004 to December 2006. These patients had previously submitted to electroencephalography (EEG), cranial computed tomography (CT), and brain magnetic resonance imaging (MRI) as part of routine investigations for epilepsy.

The interview was directed with special attention to the use of medications and the presence of autonomic symptoms and signs, such as sweating, nausea, pallor, dizziness, sphincter release, muscle contractions, drowsiness, and confusion $^{11}$. The questionnaire of the European Society of Cardiology International Study on Syncope of Uncertain Etiology 2 (ISSUE 2) ${ }^{12}$ was used with some adaptations: the age for the original inclusion was more than 30 years old, and in our group was more than 16 years old; and the adenosine triphosphate (ATP) test was replaced with the IT.

The search for signs and symptoms related to subclavian steal syndrome was performed through anamnesis. Patients were asked about signs and symptoms related to vertebrobasilar insufficiency triggered by physical exertion with the upper limbs. The assessment of peripheral pulses and blood pressure (BP) measurement in the upper limbs were performed in all patients ${ }^{13}$. The presence of thyroid disease was investigated through clinical and physical examination. In cases with clinical suspicion of thyroid dysfunction, a dose of ultrasensitive thyroid-stimulating hormone (TSH) was administered. All of the patients submitted to blood tests, which included blood count; fasting blood glucose; and serum sodium, potassium, urea, and creatinine.

Protocol for implementation of IT: The software used to collect and analyze signals was the Biopotentials Uptake System developed by Barbosa et $\mathrm{al}^{14,15}$, which was validated for reproducibility and used in other studies ${ }^{16,17}$. The patients were instructed to avoid drinking autonomic nervous system stimulants such as coffee and tea, not to perform strength exercises, and to avoid smoking and/or taking any drugs for at least 24 hours before the test.

The IT was performed in an environment with a cardiac defibrillator and equipment necessary to perform cardiopulmonary resuscitation. The non-sensitized IT was performed in an air-conditioned (ambient temperature of $22-25^{\circ} \mathrm{C}$ ) cork-lined room between 9:12 AM and late in the afternoon after fasting for at least 4-6 hours. The EKG (with modified D1 derivation) and $\mathrm{BP}$ were recorded during the 60 minutes of the examination. The BP was monitored with a non-invasive device, with a standard adult cuff positioned $3 \mathrm{~cm}$ from the left cubital fossa and programmed to inflate/deflate every 2 minutes $^{18}$. After 20 minutes in the supine position, the patients were passively tilted to an angle of $70^{\circ}$, where they remained for 40 minutes.

Protocol for the carotid sinus massage (CSM): The CSM protocol was only performed in the tilt position, during the 
last 5 minutes of the IT. It was first carried out on the right, followed by the left if the right side was negative. Pressure was exerted with a longitudinal movement at the point where the maximum carotid pulse was palpated, directly above the thyroid cartilage and below the angle of the jaw, for a period of 10 seconds. Patients who had asystole greater than or equal to 3 seconds were classified as having carotid sinus hypersensitivity in a cardioinhibitory form. Patients who exhibited a BP decrease of $5 \mathrm{mmHg}$ were classified as having hypersensitivity in a vasodepressant form. If both responses were present, the diagnosis was positive for the mixed form ${ }^{19}$.

After completion of the maneuver, the patients were asked about the presence of symptoms during and after the procedure.

Video-EEG monitoring: Surface video-EEG monitoring was performed in all patients, using a 24-channel Neuromap ${ }^{\circledR}$ NEUROTEC $^{\circledR}$ digital apparatus with the 10-20 international electrode placement system. During monitoring, the patients were submitted to activations, such as hyperventilation for 3 minutes and intermittent photostimulation.

The variables studied were recorded in Microsoft Excel ${ }^{\circledR}$, and the resulting spreadsheet was converted into a database file and read with the program Epi Info ${ }^{\circledR}$, version 6.0. The frequencies of the variables found are shown as percentages. Patients with positive or negative IT were divided into two groups for analysis of associated variables, and the differences between values were analyzed with Student's $t$-tests. A difference was considered significant when $\mathrm{p}<0.05$.

\section{RESULTS}

Among the 35 included patients, 19 were female (54.3\%). The mean age of the patients was 35.0 years (range 16.0 to 61.0 years), and their mean age at the onset of events diagnosed as epileptic was 14.8 years (range 0.4 to 52.0 years, standard deviation=2.34). The mean disease duration was 22.6 years (range 4 to 47 years). Eighteen patients (51.4\%) were using 3 AEDs, 13 (37.2\%) were using 2 AEDs, and 4 patients (11.4\%) were using 4 AEDs. Six (17.1\%) patients had a diagnosis of PGS, and 29 (82.9\%) were diagnosed with SGS.

Blood tests, including blood count, blood glucose, and electrolytes were normal in all cases. A patient with thyroid swelling was assessed with specific tests that showed hypothyroidism. This patient had epilepsy with EEG-relevant changes, and the IT was positive.

CSM was negative in all patients. Interictal EEG was normal in $10(28.6 \%)$ patients. Of the $25(71.4 \%)$ patients with abnormal EEG, 21 showed focal changes (84.0\%) and epileptiform discharges (sharp waves and/or spikes, followed or not by slow waves) had temporal projections in 15 (60.0\%).

A cranial CT scan was performed in 34 patients and was normal in 26 (76.4\%). The CT scan was unable to discriminate between patients with positive versus negative IT ( $\mathrm{p}=0.10)$.

Brain MRI was performed in $33(94.2 \%)$ patients and was abnormal in 19 (57.5\%). The most frequent abnormality was hippocampal atrophy, which was found in seven cases (36.8\%). Six patients (31.5\%) had vascular malformations, and six (31.5\%) had several changes (e.g., cystic lesions, cortical dysplasia, and schizencephaly). The figure shows the results for the 35 patients who underwent the IT.

We identified nine $(25.7 \%)$ patients with positive IT. Five of them presented autonomic symptoms, one with postural orthostatic tachycardia (POTS), and four with vasovagal syncope (VVS). Seven (77.7\%) patients had physiological pseudoseizures (PPS), and 2 (22.2\%) experienced psychogenic seizures (PS). Of the seven with PPS, five (55.5\%) had a vasovagal response and two $(22.2 \%)$ had POTS. Three $(33.3 \%)$ patients received a comorbidity diagnosis of epilepsy and syncope (Table 1).

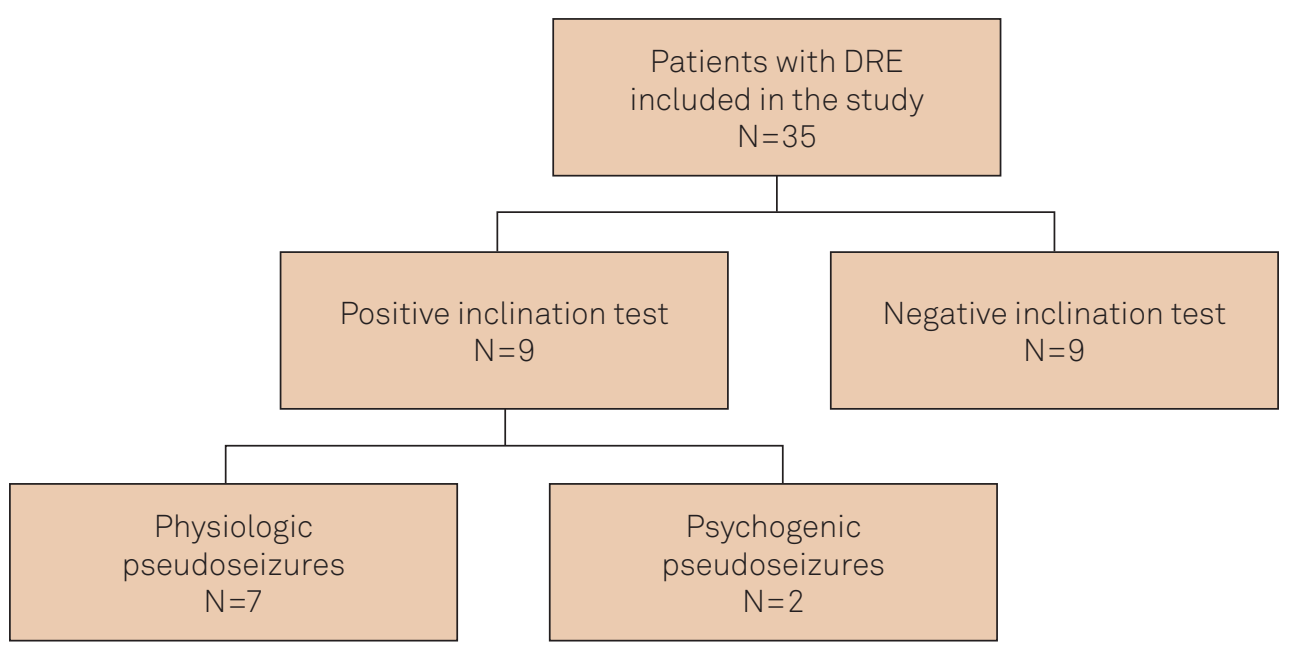

Abbreviations: DRE: drug-resistant epilepsy.

Figure. Distribution of outcomes for 35 patients who completed inclination tests (ITs). 
Table 1. Frequency of autonomic symptoms in positiveIT patients and the relationship with physiological pseudoseizures, psychogenic seizures, and comorbidity of epilepsy and syncope.

\begin{tabular}{|c|c|c|c|c|}
\hline & $\begin{array}{c}\text { Tilt } \\
\text { positive } \\
(n=9) \\
25.7 \%\end{array}$ & $\begin{array}{l}\text { PPS } \\
(n=7) \\
77.8 \%\end{array}$ & $\begin{array}{c}\text { PS } \\
(n=2) \\
22.2 \%\end{array}$ & $\begin{array}{c}\text { Comorbidity (epilepsy } \\
\text { and syncope) } \\
\qquad \begin{array}{c}(n=3) \\
33.3 \%\end{array}\end{array}$ \\
\hline POTS & $1(11.1 \%)$ & $2(22.2 \%)$ & 0 & 0 \\
\hline VVS & $4(44.4 \%)$ & $5(55.5 \%)$ & 0 & $3(33.3 \%)$ \\
\hline
\end{tabular}

IT: inclination test; OS: psychogenic seizures; POTS: postural orthostatic tachycardia; PPS: physiological pseudoseizures; VVS: vasovagal syncope.

Table 2. Analysis of the demographic and clinical variables of patients with positive and negative IT.

\begin{tabular}{|c|c|c|c|}
\hline & $\begin{array}{l}\text { Tilt positive } \\
\quad(n=9) \\
25.7 \%\end{array}$ & $\begin{array}{l}\text { Tilt negative } \\
\qquad(n=26) \\
(74.3 \%)\end{array}$ & $p$ \\
\hline Gender & & & 0.01 \\
\hline Male & $1(11.1 \%)$ & $15(57.7 \%)$ & \\
\hline Female & $8(89.9 \%)$ & $11(42.3 \%)$ & \\
\hline Race & & & 0.28 \\
\hline Caucasian & $7(77.8 \%)$ & $15(57.7 \%)$ & \\
\hline Afro-descendants & $2(22.2 \%)$ & $11(42.3 \%)$ & \\
\hline Type of seizure & & & 0.63 \\
\hline Focal & $7(77.8 \%)$ & $22(84.6 \%)$ & \\
\hline Generalized & $2(22.8 \%)$ & $4(15.4 \%)$ & \\
\hline EEG & & & 0.08 \\
\hline Normal & $4(44.4 \%)$ & $6(23.1 \%)$ & \\
\hline Abnormal & $5(56.6 \%)$ & 20 (76.9\%) & \\
\hline MRI & & & 0.08 \\
\hline Normal & $6(66.7 \%)$ & $8(30.8 \%)$ & \\
\hline Abnormal & $3(33.3 \%)$ & $16(61.5 \%)$ & \\
\hline
\end{tabular}

EEG: electroencephalogram; IT: inclination test; MRI: magnetic resonance imaging.

We found a significant association between autonomic symptoms and a positive IT $(\mathrm{p}=0.005)$. Among the nine patients with a positive IT, five (55.5\%) had autonomic symptoms (one patient with POTS and four with VVS). The two patients with a positive response in IT psychogenic syncope underwent video-EEG and showed no electroclinical correlation seizures, confirming the diagnosis of pseudoseizures. The use of AEDs showed no statistically significant association with the presence of syncope.

Table 2 shows the analysis of demographic and clinical variables in patients with positive and negative IT results.

\section{DISCUSSION}

One of the major difficulties of diagnosing epilepsy is distinguishing among true seizures, syncope and PS 9 . Our findings are strong evidence of this difficulty in diagnosing DRE in patients referred to an Epilepsy Program at an University
Hospital. This was demonstrated by the mixed hemodynamic vasovagal and psychogenic response types in the IT. Our rate of $25.7 \%$ of patients with non-epileptic events is similar to data presented by Smith et $\mathrm{al}^{20}$, who were unable to confirm the diagnosis of DRE in 46 (25\%) of 184 of patients referred for treatment at a specialized clinic. Likewise, Mory et al. ${ }^{21}$ reviewed 41 cases of patients with focal epilepsy who were followed in a specialized epilepsy center and showed that 25 received an incorrect diagnosis of epilepsy. A lack of pathophysiological understanding, inexperience of the examiner, and difficulty obtaining an adequate clinical history to detect other conditions that may mimic an epileptic seizure can complicate the diagnostic process ${ }^{22}$. Another important factor is the overvaluation of examinations, especially the EEG, which may be abnormal in patients without epilepsy ${ }^{22}$.

One of our patients had a diagnosis of PS and started psychotherapy and antipsychotic drugs. He experienced a significant reduction in the paroxysmal events previously diagnosed as DRE that were in fact not epileptic. We consider our findings important because they allowed a review of the change in diagnosis and treatment planning. Several studies point to the importance of the differential diagnosis between epilepsy and PS, allowing changes in the workup and treatment ${ }^{8,23}$. We consider it important to emphasize some clinical data for suspected PS, such as fixed triggering affective factors, patients extremely worried or paradoxically indifferent about their symptoms, and crisis never witnessed.

Five patients had a diagnosis of VVS. Our data are consistent with literature showing that VVS should be the main differential feature from PPS in patients with DRE. As shown in a previous study ${ }^{24}$, all of our patients with VVS exhibited improvement of "seizures" with specific treatment, and when most AEDs had been suspended. All our patients with syncope and a diagnosis of PPS had a satisfactory response to the treatment for syncope, except one who had POTS and concomitant epilepsy.

We identified autonomic symptoms in 8 of 35 patients (22.8\%), which they attributed to their seizures. We found that the presence of autonomic symptoms was conditional on a positive IT $(p<0.005)$.This strong association indicates an important role in the differential diagnosis of dysautonomia in patients with DRE. We found no difference in focal or generalized seizure semiology between the two groups. Kim et al. ${ }^{25}$ showed that approximately two-thirds of patients with a vasovagal response also had autonomic prodromal symptoms.

Of the three patients who had a concomitant diagnosis of DRE and syncope, none had syncope suspected initially. Our results are consistent with those of Smith ${ }^{9}$, who considered that it was most difficult to distinguish epileptic seizures from syncope and psychogenic attacks. Several studies demonstrated that they frequently coexist, and attributed this to the high prevalence of these conditions in the general population. Of these three patients, two experienced significant 
seizure reduction with the treatment of syncope: they were able to go for a few months without experiencing crisis.

We recorded abnormal EEG results in 25 (71.4\%) patients. The most frequent changes were ictal temporal patterns in $42 \%$ of patients with an abnormal EEG. We correlated these results to the MRI findings of our patients, and the most frequent abnormality was mesial hippocampal atrophy (36.8\%). Studies in adults show that epileptiform interictal activity is found in $29-55 \%$ of epileptic patients in the first EEG. If the origin of epilepsy involves deeper structures, the EEG may be normal in $60-70 \%$ of patients ${ }^{26}$. Our group had a higher percentage of abnormal EEGs, probably because this study assessed patients with DRE. Despite the limitations of the interictal EEG, it is considered a very useful diagnostic test because it can corroborate the diagnosis of epilepsy, and it provides valuable information about epilepsy type and prognosis ${ }^{26}$.

When we examined interictal EEG findings in patients with syncope, we found that $56.6 \%$ had abnormal EEGs. We expected to find a higher frequency of normal EEGs in patients with syncope, but no difference was found between normal and abnormal EEGs in groups of patients with and without syncope ( $\mathrm{p}=0.08$ ). A study by Reuber et al. ${ }^{27}$ investigated interictal EEG abnormalities in patients with non-epileptic PS and showed electrographic abnormalities in $18 \%$ of cases, with no other associated pathology. As we only had two cases of non-epileptic PS, we cannot compare our data with those of Reuber et $\mathrm{al}^{27}$. However, our findings show that EEG findings are not a useful parameter for the differential diagnosis of syncope. Our results are consistent with the literature, which demonstrates that it is possible to find EEG changes considered epileptiform in non-epileptic patients with clinical symptoms of syncope, leading to misdiagnosis and unnecessary treatment ${ }^{26}$. Toledo ${ }^{28}$ also reported two cases of convulsive syncope during the implantation of sphenoidal electrodes in epileptic patients.

Other diseases can mimic epilepsy, leading to the misdiagnosis of DRE. These include insulinoma ${ }^{29}$, cardiogenic syncope $^{24}$, and carotid sinus hypersensitivity ${ }^{19}$. On the other hand, there are reported cases of ictal bradycardia or tachyarrhythmia during seizures, causing more diagnostic confu$\operatorname{sion}^{30}$. Linzer et al. ${ }^{30}$ published a series of 12 cases diagnosed as epileptic for a long period, and 11 were treated with AEDs. These patients were subsequently diagnosed as suffering from arrhythmia or VVS. Collectively, the literature confirms that history and physical examination are essential for a differential diagnosis between epilepsy and other diseases in which neurological symptoms occur, and should not be substituted by any further examination. Reuber et al. ${ }^{27}$ showed EEG changes in many patients without a diagnosis of epilepsy, including epileptiform changes. Although prolonged video-EEG monitoring with surface electrodes is a mandatory test for the diagnostic investigation of DRE, it may fail in some cases, and the use of deep electrodes is sometimes needed ${ }^{26}$. We showed that the best way to differentiate between DRE and syncope was to assess the anamnesis data. The studies discussed here concluded that a detailed medical history with thorough descriptions of events is the main tool for distinguishing between syncope and epilepsy ${ }^{30}$.

In conclusion, we found a significant frequency of syncope in our population of patients diagnosed with DRE without apparent heart disease. EEG and MRI and CT scans did not influence the differential diagnosis or the suspicion of the diagnosis of syncope in this population. Autonomic manifestation was the most important clinical phenomenon and was significantly associated with the group of patients with syncope $(\mathrm{p}<0.05)$, surpassing the importance of examinations in contributing to the differential diagnosis of patients with DRE. Although prolonged video-EEG monitoring is the most relevant diagnostic test for investigating DRE, the association with the IT is very useful to establish the differential diagnosis of syncope, and this should be routinely performed in patients diagnosed with DRE who present with autonomic symptoms.

The main finding of this study can contribute significantly to the management of patients with DRE. The identification of autonomic symptoms associated with a positive IT in patients diagnosed with DRE and without apparent heart disease can target this group of patients for more thorough examination. Misdiagnosis has severe consequences for patients, including decreased quality of life; social, psychological, and behavioral problems; and the possibility of side effects from unnecessary exposure to AEDs. Our findings have important clinical implications that may facilitate changes in therapy.

\section{References}

1. Carpio A, Hauser WA. Epilepsy in the developing world. Curr Neurol Neurosci Rep 2009;9:319-326.

2. Birbeck GL. Revising and refining the epilepsy classification system: priorities from a developing world perspective. Epilepsia 2012;53(Suppl 2):S18-S21.

3. Nadkarni S, Lajoie J, Devinsky O. Current treatments of epilepsy. Neurology 2005;64 (Suppl 3):S2-S11.
Velasco TC, Wichert-Ana L, Walz R. Video-EEG monitoring in patients with normal MRI. Arq Neuropsiquiatr 2003;61(Suppl 1);S65-S71.

Devinsky O. Current concepts: patients with refractory seizures. N Engl J Med 1999;340:1565-1570.

Goldschlager N, Epstein A, Grubb B, et al. Etiologic considerations in the patient with syncope and an apparently normal heart. Arch Intern Med 2003;163:151-162. 
Smars PA, Decker WW, Shen WK. Syncope evaluation in the emergency department. Cur Opin Cardiol 2007;22:44-48.

8. Zaidi A, Clough P, Cooper P, et al. Misdiagnosis of epilepsy: many seizure-like attacks have a cardiovascular cause. J Am Coll Cardiol 2000;36:181-184.

9. Smith PEM. If it's not epilepsy. J Neurol Neurosurg Psychiatry 2001;70(Suppl 2):S9-S14.

10. Engel $\mathrm{J} \mathrm{J}$ r. Report of the ILAE classification core group. Epilepsia 2006;47:1558-1568.

11. Kapoor WN, Karpf M, Wieand S, et al. A prospective evaluation and follow-up of patients with syncope. $N$ Engl $J$ Med 1983;309:197-204.

12. The Steering Committee of the ISSUE 2 study. The International Study on Syncope of Uncertain Etiology 2: the management of patients with suspected or certain neutrally mediated syncope after the initial evaluation rationale and study design. Europace 2003; 5:317-321.

13. Fregni B, Branco LECC, Conforto AB, et al. Treatment of subclavian steal case report. Arq Neuropsiquiatr 2003;61:95-99.

14. Barbosa PRB, Barbosa Filho J, Cordovil I, Medeiros AB, Nadal J. Phase response of the spectral coherence between heart rate variability and ventricular repolarization duration in normal subjects. Comput Cardiol 2000;27:159-162.

15. Barbosa Filho J, Barbosa PR, Cordovil I. Autonomic modulation of the heart in systemic arterial hypertension. Arq Bras Cardiol 2002;78:181-195.

16. Bittencourt MI, Barbosa PR, Drumond Neto C, et al. Assessing autonomic function in hypertrophic cardiomyopathy. Arq Bras Cardiol 2005;85:388-396.

17. Portella RB, Pedrosa RC, Coeli CM, Buescu A, Vaisman M. Altered cardiovascular vagal responses in nonelderly female patients with subclinical hyperthyroidism and no apparent cardiovascular disease. Clin Endocrinol 2007;67:290-294.

18. Sutton R, Bloomfield DM. Indications, methodology, and classification of results of tilt-table testing. Am J Cardiol 1999;84:10-19.
19. Benchimol M, Souza RO. Syncope in the elderly: diagnostic utility of carotid sinus massage in the head-up tilt test. Arq Neuropsiquiatr 2003;61:87-90.

20. Smith D, Defalla BA, Chadwick DW. The misdiagnosis of epilepsy and the management of refractory epilepsy in a specialist clinic. Q J Med 1999;92:15-23.

21. Mory SB, Guerreiro CAM, Min LL, Teixeira RA, Costa AL, Cardoso TA, Cendes F. Idiopathic generalized epilepsies misdiagnosed as partial epilepsies. Arq Neuropsiquiatr 2002;60:788-796.

22. Thijs RD, Wieling W, Kaufmann H, van Dijk G. Defining and classifying syncope. Clin Auton Res 2004;14(Suppl 1):S4-S8.

23. Marques LHN, Almeida SJA, Santos AB. Monitorização vídeoEEG prolongada em crises não epilépticas. Arq Neuropsiquiatr 2004;62:463-468.

24. Bergfeldt L. Differential diagnosis of cardiogenic syncope and seizure disorders. Heart 2003;89:353-358.

25. Kim KH, Cho JG, Lee KO et al. Usefulness of physical maneuvers for prevention of vasovagal syncope. Circ J 2005;69:1084-1088.

26. Cosenza-Andraus ME, Nunes-Cosenza CA, Gomes-Nunes R, Fantezia-Andraus C, Alves-Leon SV. Monitorización prolongada por videoelectroencefalagrafia de pacientes com diagnóstico ambulatório de epilepsia del lóbulo temporal de difícil control: aplicación del modelo de lógica fuzzy. Rev Neurol 2006;43:7-14.

27. Reuber M, Fernandez G, Bauer J, et al. Interictal EEG abnormalities in patients with psychogenic nonepileptic seizures. Epilepsia 2002;43:1013-1020.

28. Toledo JC. Convulsive syncope following placement of sphenoidal electrodes. Brain Dev 1999;21:213-215.

29. Graves TD, Gandhi S, SMITH SJ et al. Misdiagnosis of seizures: insulinoma presenting as adult-onset seizure disorder. J Neurol Neurosurg Psychiatry 2004;75:1091-1092.

30. Linzer M, Grubb BP, Ho S, et al. Cardiovascular causes of loss of consciousness in patients with presumed epilepsy: a cause of the increased sudden death rate in people with epilepsy? Am J Med 1994;96:146-154. 